\title{
Quantum Hall Ferromagnets
}

\author{
Tin-Lun Ho \\ 1 Physics Department, The Ohio State University, Columbus, Ohio 43210 \\ National High Magnetic Field Laboratory, Florida State University, Tallahassee, FL 32306-4005
}

\begin{abstract}
It is pointed out recently that the $\nu=1 / m$ quantum Hall states in bilayer systems behave like easy plane quantum ferromagnets. We study the magnetotransport of these systems using their "ferromagnetic" properties and a novel spin-charge relation of their excitations. The general transport is a combination of the ususal Hall transport and a time dependent transport with quantized time average. The latter is due to a phase slippage process in spacetime and is characterized by two topological constants.
\end{abstract}

\section{PACS no. i75.10.-b, 73.20.Dx, 64.60.Cn}

Recent technological advances in producing high mobility bilayers have provided opportunities for studying quantum Hall (QH) systems with internal degrees of freedom [3]. In this case, the internal degrees of freedom is a pseudo-spin 1/2 labelling the upper and lower layer. There are experimental evidence 傗 indicating that under appropriate conditions, $\mathrm{QH}$ states (at $\nu=1 / 2$ and $\nu=1$ ) can be stabilized by correlations between layers and have little to do with tunneling between them. It is conceivable that similar correlated states will be found at lower filling factors. Bilayer $\mathrm{QH}$ states with $\nu=1 / m$, ( $\mathrm{m}$ odd) are believed to be the so-called $(\mathrm{mmm})$ state. It is realized recently that they are very unusual QH fluids. Wen and Zee [5] showed that these states can produce Josephson like tunneling between the layers. Very recently, a number of authors [2] have pointed out that these states behave like easy plane quantum ferromagnets and have studied possible phase transitions in these systems.

The purpose of this paper is to study the magnetotransport of QH ferromagnets (QHFs). The difficulty of the study arises from the fact that the usual procedure of extracting conductivity tensor (say, using flux insertion arguments or Chern-Simon type of theories) breaks down when applied to the $(\mathrm{mmm})$ state [6]. Here, we take a new approach which amounts to studying the hydrodynamics of QHFs. Our approach recovers the known results for ordinary QH fluids, and reveals a new transport mode in QHF. A general QHF transport is a combination of usual QH trasnport and a genuinely time dependent transport with quantized time average. The latter corresponds to a phase slippage process which turns out to be a continuous nucleation of coreless vortices in spacetime. The conducitivity tensor for the time average currents is characterized by two topological constants specifying the nucleation process. It is interesting to note that there is a close analogy between QHF and superfluid ${ }^{3} \mathrm{He}-\mathrm{A}$. They have similar order parameters, topological excitations, and vortex nucleation processes.

To determine the magnetotransport of a bilayer system, we need to find the current densities $\mathbf{J}_{\mu}$ for given electric fields $\mathbf{E}_{\mu}$ in each layer, where $\mu=\uparrow$ and $\downarrow$ denote the upper and lower layer respectively. A general configuration $\left\{\mathbf{E}_{\mu}\right\}$ is a sum of identical and opposite fields $\left(\mathbf{E}_{\uparrow}, \mathbf{E}_{\downarrow}\right)=\mathbf{E}_{+}(1,1)+\mathbf{E}_{-}(1,-1) ; \mathbf{E}_{ \pm}=\frac{1}{2}\left(\mathbf{E}_{\uparrow} \pm \mathbf{E}_{\downarrow}\right)$. The cases $\left(\mathbf{E}_{+} \neq 0, \mathbf{E}_{-}=0\right)$ and $\left(\mathbf{E}_{+}=0, \mathbf{E}_{-} \neq 0\right)$ will be referred to as the "in-phase" and "out-of-phase" mode. Their corresponding current responses will be denoted by a subscript "+" and "-" respectively. In linear response, it is sufficient to consider these modes separately. The "in-phase" mode is the usual QH transport, with current response

$$
\left(\mathbf{J}_{\uparrow}+\mathbf{J}_{\downarrow}\right)_{+}=\nu \frac{e^{2}}{h} \hat{\mathbf{B}} \times \mathbf{E}_{+}, \quad\left(\mathbf{J}_{\uparrow}-\mathbf{J}_{\downarrow}\right)_{+}=0
$$

where $\hat{\mathbf{B}}$ is the direction of the magnetic field, taken to be normal to the layers in our discussions. The "out-ofphase" mode is unique to bilayer systems. For QHFs, we shall show that d.c.fields $\mathbf{E}_{-}$will generate time dependent currents. The sum $\left(\mathbf{J}_{\uparrow}+\mathbf{J}_{\downarrow}\right)_{-}$oscillates with period $T=2 T_{o}$, where $T_{o}$ is the Josephson period $e \Delta V / \hbar$, and $\Delta V$ is the voltage that generates $\mathbf{E}_{-}$. The factor of 2 is a reflection of the double-valueness of the pseudo-spin $1 / 2$. The time average current response (denoted by a "bar") can be determined from general argument and are given by

$$
\overline{\left(\mathbf{J}_{\uparrow}+\mathbf{J}_{\downarrow}\right)_{-}}=n_{A} \nu \frac{e^{2}}{h} \hat{\mathbf{B}} \times \mathbf{E}_{-}, \overline{\left(\mathbf{J}_{\uparrow}-\mathbf{J}_{\downarrow}\right)_{-}}=n_{B} \frac{\nu}{2} \frac{e^{2}}{h} \hat{\mathbf{B}} \times \mathbf{E}_{-},
$$

where $n_{A}$ and $n_{B}$ are integers. By studying a number of examples consistent with the driving force $\mathbf{E}_{-}$, we find $n_{A}=0, n_{B}=1$. The sum of eq.(11) and (2) then gives

$$
\overline{\left(\begin{array}{c}
\mathbf{J}_{\uparrow} \\
\mathbf{J}_{\downarrow}
\end{array}\right)}=\nu \frac{e^{2}}{8 h}\left(\begin{array}{ll}
3 & 1 \\
1 & 3
\end{array}\right) \hat{\mathbf{B}} \times\left(\begin{array}{l}
\mathbf{E}_{\uparrow} \\
\mathbf{E}_{\downarrow}
\end{array}\right)
$$

These examples also reveal an interesting oscillatory behavior of the currents. The time average of $\left(\mathbf{J}_{\uparrow}+\mathbf{J}_{\downarrow}\right)_{-}$ over the half period $T_{o}$ is $\pm \nu\left(e^{2} / h\right) \hat{\mathbf{B}} \times \mathbf{E}_{-}$, with alternating sign every half period. The time average of $\left(\mathbf{J}_{\uparrow}-\mathbf{J}_{\downarrow}\right)_{-}$ is $(\nu / 2)\left(e^{2} / h\right) \hat{\mathbf{B}} \times \mathbf{E}_{-}$for all half periods. 
To derive these results, we recall that the Hamiltonian of the bilayer system in the weak tunneling limit is 7

$$
\begin{aligned}
& H=\frac{1}{2 m} \int d \mathbf{r}\left[\hat{\psi}^{+}\left(\mathbf{p}-\frac{e}{c} \mathbf{A}\right)^{2} \hat{\psi}-\Delta \hat{m}_{1}(\mathbf{r})\right] \\
& +\frac{1}{2} \int\left[\hat{\rho}(\mathbf{r}) \hat{\rho}\left(\mathbf{r}^{\prime}\right) v\left(\mathbf{r}-\mathbf{r}^{\prime}\right)+\hat{m}_{3}(\mathbf{r}) \hat{m}_{3}\left(\mathbf{r}^{\prime}\right) v_{1}\left(\mathbf{r}-\mathbf{r}^{\prime}\right)\right]
\end{aligned}
$$

where $\mathbf{r}=(x, y), \hat{\psi}_{\mu}(\mathbf{r})$ creates an electron at the $\mu$ layer at $\mathbf{r}, \hat{m}_{i}=\frac{1}{2} \operatorname{Tr} \hat{\psi}^{+} \sigma_{i} \hat{\psi},(i=1,2,3), \hat{\rho}=\operatorname{Tr} \hat{\psi}^{+} \hat{\psi}$ and both $v, v_{1}>0$. The pseudo-spin axes $\hat{\mathbf{x}}_{1}, \hat{\mathbf{x}}_{2}, \hat{\mathbf{x}}_{3}$ are chosen so that the symmetric and antisymmetric state (with energy difference $\Delta)$ are represented by $\left(\begin{array}{l}1 \\ 1\end{array}\right)$ and $\left(\begin{array}{l}1 \\ -1\end{array}\right)$. They have no relations with the real space directions $\hat{\mathbf{x}}, \hat{\mathbf{y}}, \hat{\mathbf{z}}$.

We shall consider the $(\mathrm{mmm})$ state in the correlation limit $\Delta<<e^{2} / \epsilon_{o} \ell \sim e^{2} / \epsilon_{o} d$, where $d$ is the spacing between layers, and $\epsilon_{o}$ is the dielectric constant. We begin by expressing their wavefunctions (in the symmetric gauge) in spinor form

$$
\Psi\left(\begin{array}{l}
\mathbf{r}_{1} \\
\mu_{1}
\end{array}, \ldots \mu_{N} \mathbf{r}_{N}\right)=e^{-\sum_{i=1}^{N}|z|_{i}^{2} / 4} \prod_{i=1}^{N} \zeta_{\mu_{i}} \prod_{i>j}\left(z_{i}-z_{j}\right)^{m},
$$

where $z=x+i y$ and

$$
\zeta=\left(\begin{array}{l}
u \\
v
\end{array}\right)=|\zeta|\left(\begin{array}{l}
e^{-i \phi / 2} \cos \theta / 2 \\
e^{i \phi / 2} \sin \theta / 2
\end{array}\right) e^{-i \chi / 2}
$$

is a spinor along $\hat{\ell}=\hat{\mathbf{x}}_{3} \cos \theta+\sin \theta\left(\hat{\mathbf{x}}_{1} \cos \phi+\hat{\mathbf{x}}_{2} \sin \phi\right)$. Since $v_{1}>0$, it is energetically more favorable for $\hat{\ell}$ to lie in the $\mathrm{x}_{1}-\mathrm{x}_{2}$ plane. $\Delta$ will line up $\hat{\ell}$ along $\hat{\mathbf{x}}_{1}$. However, in the correlation limit, it remains small compared to the correction energy. The symmetric and antisymmetric states can therefore be regarded as essentially degenerate - - a view that we shall adopt in our subsequent discussions for simplicity [8].

If uniform potentials $V_{\uparrow}$ and $V_{\downarrow}$ are applied to in the upper and lower layer, $u$ and $v$ will evolve as $e^{i e V_{\uparrow} t / \hbar}$ and $e^{i e V_{\downarrow} t / \hbar},(e>0)$, meaning

$$
-\partial_{t} \phi=\frac{e}{\hbar}\left(V_{\uparrow}-V_{\downarrow}\right) \equiv \frac{e V}{\hbar}, \quad-\partial_{t} \chi=\frac{e}{\hbar}\left(V_{\uparrow}+V_{\downarrow}\right) \equiv \frac{2 e U}{\hbar} .
$$

Eq.(7) implies means that $\hat{\ell}$ precesses about $\hat{\mathbf{x}}_{3}$ with angular frequency $e V / \hbar$. This immediately implies an "unstable" situation if $V$ is different at two points far apart, (i.e. $\mathbf{E}_{-} \neq 0$ ). The $\hat{\ell}$ vectors at these points will assume their ground state configurations, (lying in $\mathrm{x}_{1}-\mathrm{x}_{2}$ plane) but rotate about $\hat{\mathbf{x}}_{3}$ at different rates. As a result, more and more winding in the spin texture is produced which can only be relieved by phase slippage processes. As we shall see, during phase slippage, $\hat{\ell}$ will be pulled away from the $\mathrm{x}_{1}-\mathrm{x}_{2}$ plane every now and then in some region in space. While this costs nucleation energy because of the last term in eq.(4), it can not be stopped because its energy cost will evenetually be surpassed by the gradient energy generated by $\mathbf{E}_{-}$.
Nonuniform textures implies spatially varying spinors. However, to stay in the lowest Landau, $\zeta$ must be analytic in $z$, (or a function of $\partial_{z}$ ). For simplicity, we shall from now on focus on the "quasihole" case where $\zeta(z)$ is an analytic function of $z$. In general, $\zeta$ can be expanded in powers of $z$. The simplest example of a nonuniform analytic spinor is $\zeta(z)=\alpha+\beta z$, where $\zeta$ reduces to spinors $\alpha$ and $\beta$ as $z$ approaches 0 and $\infty$. It also reduces to the usual quasihole excitations if $\alpha \propto \beta$. For arbitrary $\zeta$, eq.(E) implies that

$$
2 \mathbf{m}(\mathbf{r})=\rho(\mathbf{r}) \hat{\ell}(\mathbf{r})
$$

This is a statement of "full" ferromagnetism and is important for our subsequent discussions.

The relations between density and spin fluctation can be obtained from the "plasma analog" [9], where one interprets $\sum_{[\mu]}|\Psi([\mathbf{r}, \mu])|^{2}$ as the partition function of a classical 2D plasma with charge $m$ in a background potential $V_{b}(z)=-|z|^{2} / 2+\ln |\zeta|^{2}$. This implies that the electron density is $\rho(\mathbf{r})==-(4 \pi m)^{-1} \nabla^{2} V_{b}$. $=$ $(2 \pi m)^{-1}-(4 \pi m)^{-1} \nabla^{2} \ln |\zeta|^{2}$. Because of the analyticity of $\zeta$, the density change can be expressed in terms of a deformation field $[10] \mathbf{u}=-i \zeta^{+} \nabla \zeta /|\zeta|^{2}+$ c.c.

$$
\rho(\mathbf{r})=\frac{1}{2 \pi m}-\frac{1}{4 \pi m} \hat{\mathbf{z}} \cdot \nabla \times \mathbf{u}
$$

If follows from eq.(6) that 11] [12

$$
\hat{\mathbf{z}} \cdot \nabla \times \mathbf{u}=-(\hat{\mathbf{z}} \times \nabla) \cdot \nabla \chi+\frac{1}{2} \epsilon_{\alpha \beta \gamma} \ell_{\alpha}(\hat{\mathbf{z}} \times \nabla) \ell_{\beta} \cdot \nabla \ell_{\gamma} .
$$

The first term vanishes unless $\chi$ is a vortex, in which case it is a $\delta$-function 13 .

The total electric current $\mathbf{J}=\mathbf{J}_{\uparrow}+\mathbf{J}_{\downarrow}$ can be obtained by differentiating the number density eq.(9) and using the continuity equation $(-e) \dot{\rho}=-\nabla \cdot \mathbf{J}$,

$$
J_{\alpha}=\frac{1}{m} \frac{e^{2}}{h}(\hat{\mathbf{z}} \times \nabla)_{\alpha} U-\frac{e}{4 \pi m} \hat{\ell} \cdot(\hat{\mathbf{z}} \times \nabla)_{\alpha} \hat{\ell} \times \partial_{t} \hat{\ell}
$$

where we have made use eq.(7). The first term is the usual QH transport, eq.(1). (Note that $\mathbf{E}_{+}=-\nabla U$ and that eq.(5) is derived for $\hat{\mathbf{B}}=-\hat{\mathbf{z}})$. The second term is due to the motion of the spin texture, which is generated by $\mathbf{E}_{-}$. To simplify discussions and formulas, we shall from now on focus on the out-of-phase mode, i.e. $\nabla U=$ $0, \nabla V \neq 0$. We shall then drop the first terms in eq. 111) and eq.(10).

Consider now the time average of the current in $y$ over a period $T, \overline{\left(I_{\uparrow}+I_{\downarrow}\right)_{y}}=T^{-1} \int_{0}^{T} d t \int J_{y} d x$. The integral is evaluated for an arbitrarily $y$ coordinate, with upper and lower limit $x_{a}$ and $x_{b}$ denoting the edges of the sample. From eq.111 we have

$$
\overline{\left(I_{\uparrow}+I_{\downarrow}\right)_{y}}=\frac{-e}{4 \pi m} \frac{1}{T}\left[\int_{0}^{T} d t \int_{x_{a}}^{x_{b}} d x \hat{\ell} \cdot \partial_{x} \hat{\ell} \times \partial_{t} \hat{\ell}\right]
$$


The quantity in the bracket in eq.(12) (denoted as $A$ ) is the area on an unit sphere $S_{2}$ covered by the "spactime rectangle" $\Gamma$ with area $T \times L, L=\left(x_{b}-x_{a}\right)$. A nonvanishing $A$ means nucleation of vorticity in spacetime $\left[\partial_{t}(\nabla \times \mathbf{u}) \neq 0\right]$.

Eq.(12) alone cannot determine $I_{\uparrow y}$ and $I_{\downarrow y}$. From eqs.(8), (9), and (10), we have

$$
\begin{gathered}
2 \mathbf{m}(\mathbf{r})=\frac{1}{2 \pi m} \vec{\ell}-\frac{1}{4 \pi m} \partial_{x} \hat{\ell} \times \partial_{y} \hat{\ell}, \\
2 \partial_{t} \mathbf{m}=\frac{1}{2 \pi m} \partial_{t} \hat{\ell}-\frac{1}{4 \pi m} \nabla_{\alpha}\left((\hat{\mathbf{z}} \times \nabla)_{\alpha} \hat{\ell} \times \partial_{t} \hat{\ell}\right) .
\end{gathered}
$$

Eq.(14) says that spin fluctuations will generate a pseudo-spin current

$$
\overrightarrow{\mathbf{K}}_{\alpha}=\frac{1}{4 \pi m}(\hat{\mathbf{z}} \times \nabla)_{\alpha} \hat{\ell} \times \partial_{t} \hat{\ell}
$$

which is related to the charge currents as $(-e) \hat{\mathbf{x}}_{3} \cdot \overrightarrow{\mathbf{K}}_{\alpha}=$ $J_{\uparrow \alpha}-J_{\downarrow \alpha}$. The time average of current difference in $y$ is

$$
\overline{\left(I_{\uparrow}-I_{\downarrow}\right)_{y}}=\frac{-e}{4 \pi m} \frac{1}{T}\left[\int_{0}^{T} d t \int_{x_{a}}^{x_{b}} d x \hat{\mathbf{x}}_{3} \cdot \partial_{x} \hat{\ell} \times \partial_{t} \hat{\ell}\right] .
$$

The quantity in the bracket (denoted as $B$ ) is the area in $S_{2}$ covered by $\Gamma$ projected onto the $\mathrm{x}_{1}-\mathrm{x}_{2}$ plane.

To evaluate $A$ and $B$, we consider a rectangular sample (see fig.1) where the potentials $\left(V_{\uparrow}, V_{\downarrow}\right)$ at $x_{a}$ and $x_{b}$ are $(0,0)$ and $(V / 2,-V / 2)$ respectively. This is the "unstable" situation we discussed before, where $\hat{\ell}$ precesses about $\hat{\mathbf{x}}_{3}$ with angular frequency $\mathrm{eV} / \hbar$ at $x_{b}$ and remains stationary at $x_{a}$ [14]. In the steady state, the textural motion must be periodic as $\mathbf{E}_{-}$forces a constant rotation on $\zeta$ at $x_{b}$. Note that $\hat{\ell}$ at $x_{b}$ returns to itself after time $T_{o}=h / \mathrm{eV}$, whereas the period of $\zeta$ (and hence the entire texture $\hat{\ell}(x, y, t))$ is $T=2 T_{o}$. The factor of 2 is due to the double valueness of $\zeta$. An example of the steady state evolution of $\hat{\ell}$ is shown in the spacetime plot $(x-t)$ in figure 1. The evolution is a repetition of the pattern inside the rectangle $T \times L,\left(a b b^{\prime \prime} a^{\prime \prime}\right)$. Since the boundary of the rectangle maps onto the equator of $S_{2}$ twice, we then have $A=-4 \pi n_{A}, B=-2 \pi n_{B}$, where $n_{A}$ and $n_{B}$ are integers depending on how $S_{2}$ is covered. Eq.(12) and (16) then gives $\overline{\left(I_{\uparrow}+I_{\downarrow}\right)_{y}}=n_{A}(1 / m)\left(e^{2} / h\right)(V / 2)$, $\overline{\left(I_{\uparrow}-I_{\downarrow}\right)}=n_{B}(2 m)^{-1}\left(e^{2} / h\right)(V / 2)$, which is eq.(2) since $\mathbf{E}_{-}=-\frac{1}{2} \nabla\left(V_{\uparrow}-V_{\downarrow}\right)=-\frac{1}{2} \nabla V$ and $\hat{\mathbf{B}}=-\hat{\mathbf{z}}$.

To determine the topological integers $n_{A}, n_{B}$, we construct explicit examples for the out-of-phase current response. Since we are dealing with rectangular geometries, it is more convenient to use the Landau gauge. The QHF now reads [15]

$$
\Psi\left(\begin{array}{c}
{[\mathbf{r}]} \\
{[\mu]}
\end{array}\right)=e^{-\sum_{i} x_{i}^{2} / 2} \prod_{i} \zeta\left(w_{i}\right) \prod_{i>j}\left(w_{i}-w_{j}\right)^{m} .
$$

where $w=e^{\alpha z}, \alpha$ is the ratio of the magnetic length to the sample length in $\mathrm{y}$, which can be taken as 1 without loss of generality. To match the Josephson relations eq.(17) at the boundaries $x_{a}$ and $x_{b}$, (now taken to be $-\infty$ and $+\infty$ ), we choose a spinor of the form

$$
\zeta(w)=\lambda\left(\begin{array}{l}
1 \\
1
\end{array}\right)+e^{x+i y}\left(\begin{array}{c}
e^{i e V t / 2 \hbar} \\
e^{-i e V t / 2 \hbar}
\end{array}\right)
$$

where $\lambda$ is a constant setting the scale of textural destortion in real space [16]. Its textural evolution in the spacetime plane $(x-t)$ (for a given $y$ ) is shown in fig.1. $\hat{\ell}$ becomes uniform every half period $T_{o}=h / \mathrm{eV}$. The topological areas $A$ and $B$ for each half peirod can be calculated from eq.(18) and are independent of $\lambda$. $A$ is found to be $\mp 2 \pi$, with alternating signs every half period. $B$ is found to be $-\pi$ for all half periods. This means over the full period $T=2 T_{o}, A=0$ and $B=-2 \pi$, corresponding to $n_{A}=0, n_{B}=1$. We have then derived eq.(3) from this specific example. I have also examined a number of other spinors satisfying the same Josephson boundary condition at infinities. Their time average responses are identical to that of eq.(18). I therefore think that these values of $n_{A}$ and $n_{B}$ have general validity, despite the lack of a general proof which is certainly desirable.

This work was stimulated by a talk given by S. Girvin at the Aspen Center of Theoretical Physics. Various parts of this work was completed during a memorable sabbatical visit to HKUST and CUHK in Hong Kong, and an equally enjoyable visit to NHMFL at Tallahassee. I thank Nelson Cue, Kenneth Young, Hong-Ming Lai, and Bob Schrieffer for their hospitality. This work is supported by the National High Magnetic Field Laboratory.

[1] Permanant address.

[2] K. Yang, K. Moon, L. Zhang, A.H. MacDonald, S.M. Girvin, D. Yoshioka, and S.C. Zhang, to be published.

[3] Y.W. Suen, L.W. Engel, M.B. Santos, M. Shayegan, D.C. Tsui, Phys. Rev. Lett. 68, 1379 (1992); J.P. Eisenstein, G.S. Boebinger, L.N. Pfeiffer, K.W. West, adn Song He, Phys. Rev. Lett. 68, 1383 (1992).

[4] S.Q. Murphy, J.P. Einstein, G.S. Boebinger, L.N. Pfeiffer, and K.W. West, to be published.

[5] X.G. Wen and A. Zee, Phys. Rev. Lett. 69, 1811 (1992); X.G. Wen and A. Zee, Phys. Rev. B 47, 2265 (1993).

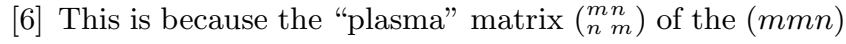
state vanishes when $m=n$.

[7] A.H. MacDonald, P.M. Platzman, and G.S. Boebinger, Phys. Rev. Lett. 65, 775, (1990).

[8] The assumption of degeneracy is not essential for our results. The derivation including non-vanishing $\Delta$ is more complicated and will be discussed elsewhere.

[9] R.B. Laughlin, Phys. Rev. Lett. 50, 1395 (1983). 
[10] When expressed in polar angles, this is exactly the superfluid velocity $\mathbf{v}_{s}$ of ${ }^{3}$ He-A. See N.D. Mermin and T.L. Ho, Phys. Rev. Lett. 36, 594, (1976), T.L. Ho, Phys. Rev. B, 18, 1144 (1978). However, unlike $\mathbf{v}_{s}, \mathbf{u}$ is invariant under time reversal.

[11] This is the vorticity equation of ${ }^{3} \mathrm{He}-\mathrm{A}$. (See N.D. Mermin and T.L. Ho, ibid; and T.L. Ho, ibid). The relation of density and spin fluctuation for polarized QH systems was pointed out by S.L. Sonhi et.al in Phys. Rev. B 47, 16419, (1993) in the context of Chern-Simon theory. It is also derived by the authors in ref.4 using projected operator methods. Our derivation here is different from these approaches. It also reveals some rather subtle identities which has significant implications on the energetics of the textural excitations. (See ref.10).

[12] Eq.(10) can be easily obtained using the identity $\zeta_{\mu} \zeta_{\nu}^{*}=$ $\frac{1}{2}\left(\delta_{\mu \nu}+\hat{\ell} \cdot \vec{\sigma}_{\mu \nu}\right)|\zeta|^{2}$. For analytic spinors, (quasihole type), their textures satisfies $(\hat{\mathbf{z}} \times \nabla)_{\alpha} \hat{\ell}=\nabla_{\alpha} \hat{\ell} \times \hat{\ell}$, which implies $\nabla_{\alpha} \ell_{\beta} \nabla_{\alpha} \ell_{\beta}=2 \hat{\ell} \cdot \partial_{x} \hat{\ell} \times \partial_{y} \hat{\ell}$. The integral $\frac{1}{2} \int d \mathbf{r}\left(\nabla_{\alpha} \hat{\ell}\right)^{2}$ is therefore a topological constant $4 \pi n$ if the texture is uniform at infinity.

[13] A singular vortex can be generated if $\zeta$ contains an usual quasihole, i.e. both $u$ and $v$ have a common factor $(z-a)$. Note that the plasma analog is only accurate on length scales larger than the magnetic length. All $\delta$-functions revealled by the plasma analog are in fact Guassians.

[14] The situation here is identical to ${ }^{3} \mathrm{He}-\mathrm{A}$ in a heat flow, where the texture is continously being wounded up by a chemical potential gradient. The winiding is undone by a periodic nucleation of coreless vortices. which is relieved by a periodic texture motion. See T.L. Ho, Phys. Rev. Lett. 41, 1473 (1978).

[15] Eq.(8) to (10) can also be derived from eq.(17). The plasma analog can be generalized easily to the Landau gauge by realizing that density of the plasma $\rho(w)$ is related to the electron density $\rho(z)$ by the Jacobian $|d w / d z|^{2}$.

[16] It follows from eq.(13) that for spinor eq.(18), $\int m_{3}=0$. This is consistent with the degenerate (or zero tunneling) limit $\Delta \rightarrow 0$ that we have taken.

\section{Figure Caption}

Figure 1 : The $\hat{\ell}$ vectors at $\mathbf{r}$ is represented by a line emerging from $\mathbf{r}$. An solid (empty) square is placed at $\mathbf{r}$ to indicate that $\hat{\ell}$ has a positive (negative) $x_{3}$ component. The boundary of the rectangle $\Gamma=\left(a b b^{\prime} a^{\prime}\right)$ maps onto the equator $\bar{\Gamma}$ on the unit sphere $S_{2}$. The lines $c \vec{c}^{\prime}, d \vec{d}^{\prime}$, $\overrightarrow{e e^{\prime}}, c^{\prime} \vec{c}^{\prime \prime}, d^{\prime} \vec{d}^{\prime \prime}$, and $e^{\prime} \vec{e}^{\prime \prime}$ map onto loops $C, D, E, \bar{C}, \bar{D}, \bar{E}$. This covering gives $A=2 \pi, B=-\pi$ for the lower rectangle $a b b^{\prime} a^{\prime}$ and $A=-2 \pi, B=-\pi$ for the upper rectangle $a^{\prime} b^{\prime} b^{\prime \prime} a^{\prime \prime}$. If the loops $\bar{C}, \bar{D}$ and $\bar{E}$ are traversed in directions opposite to those depicted, then $A=2 \pi, B=\pi$ for $a^{\prime} b^{\prime} b^{\prime \prime} a^{\prime \prime}$. The textural pattern is calculated from eq.18) with $T_{o}=1, y=2, \lambda=8$. 